\title{
On the Level Set of a Function with Degenerate Minimum Point
}

\author{
Yasuhiko Kamiyama \\ Department of Mathematics, University of the Ryukyus, Nishihara-Cho, Okinawa 903-0213, Japan \\ Correspondence should be addressed to Yasuhiko Kamiyama; kamiyama@sci.u-ryukyu.ac.jp
}

Received 14 May 2015; Accepted 21 June 2015

Academic Editor: Naseer Shahzad

Copyright (C) 2015 Yasuhiko Kamiyama. This is an open access article distributed under the Creative Commons Attribution License, which permits unrestricted use, distribution, and reproduction in any medium, provided the original work is properly cited.

For $n \geq 2$, let $M$ be an $n$-dimensional smooth closed manifold and $f: M \rightarrow \mathbb{R}$ a smooth function. We $\operatorname{set} \min f(M)=m$ and assume that $m$ is attained by unique point $p \in M$ such that $p$ is a nondegenerate critical point. Then the Morse lemma tells us that if $a$ is slightly bigger than $m, f^{-1}(a)$ is diffeomorphic to $S^{n-1}$. In this paper, we relax the condition on $p$ from being nondegenerate to being an isolated critical point and obtain the same consequence. Some application to the topology of polygon spaces is also included.

\section{Introduction and Statement of the Result}

Throughout this paper, $S^{k}$ denotes the standard topological sphere equipped with the standard differential structure. For $n \geq 2$, let $M$ be an $n$-dimensional smooth closed manifold and $f: M \rightarrow \mathbb{R}$ a smooth function. We set $\min f(M)=m$ and assume that $m$ is attained by unique point $p \in M$. Then the following result is a consequence of the Morse lemma (see, e.g., [1]): If $p$ is a nondegenerate critical point and there are no critical points in $f^{-1}((m, a])$ (where $\left.m<a\right)$, then there is a diffeomorphism $f^{-1}(a) \cong S^{n-1}$.

The purpose of this paper is to study the question of whether the same result holds if we relax the condition on $p$ from being nondegenerate to being an isolated critical point. We also give an application of our result to the topology of polygon spaces.

Now our main result is the following.

Theorem A. For $n \geq 2$, let $M$ be an $n$-dimensional smooth closed manifold and $f: M \rightarrow \mathbb{R}$ a smooth function. One sets $\min f(M)=m$ and assumes that $m$ is attained by unique point $p \in M$. If $p$ is an isolated critical point and there are no critical points in $f^{-1}((m, a])$ (where $\left.m<a\right)$, then the following results hold:

(i) If $n \neq 5$, there is a diffeomorphism $f^{-1}(a) \cong S^{n-1}$.

(ii) If $n=5$, there is a homeomorphism $f^{-1}(a) \approx S^{4}$.
Corollary B. For $n \geq 2$, let $M$ be an n-dimensional smooth closed manifold and $f: M \rightarrow \mathbb{R}$ a smooth function. One sets $\max f(M)=m$ and assumes that $m$ is attained by unique point $p \in M$. If $p$ is an isolated critical point and there are no critical points in $f^{-1}([a, m))$ (where $\left.a<m\right)$, then the following results hold:

(i) If $n \neq 5$, there is a diffeomorphism $f^{-1}(a) \cong S^{n-1}$.

(ii) If $n=5$, there is a homeomorphism $f^{-1}(a) \approx S^{4}$.

This paper is organized as follows. In Section 2 we prove Theorem A. In Section 3 we study an application of it. Theorem 4 is the main result in this section. Remark 7(ii) states the essential difference between the known map and ours.

\section{Proof of Theorem A}

We keep the notations of Theorem A. For $r \in \mathbb{R}$, we set

$$
\begin{aligned}
M^{r} & :=f^{-1}([m, r]), \\
\dot{M}^{r} & :=f^{-1}([m, r)) .
\end{aligned}
$$

Lemma 1. There is a diffeomorphism $\dot{M}^{a} \cong \mathbb{R}^{n}$.

Proof. By [2, Lemma 3], it will suffice to prove that, for any compact set $K \subset \dot{M}^{a}$, there exists an open set $V$ of $\dot{M}^{a}$ such that $K \subset V$ and $V \cong \mathbb{R}^{n}$. 
We fix an open set $U$ which satisfies that

$$
p \in U \subset \bar{U} \subset \dot{M}^{a}, \quad U \cong \mathbb{R}^{n}
$$

(Such $U$ indeed exists because $p \in \dot{M}^{a}$.) Note that $p \notin M^{a} \backslash U$ and $M^{a} \backslash U$ is compact. Note also that $\bar{U}$ is compact. Hence there exist $\varepsilon_{0}$ and $\varepsilon_{1} \in \mathbb{R}$ (where $m<\varepsilon_{0}<\varepsilon_{1}$ ) such that

$$
M^{\varepsilon_{0}} \subset U \subset \bar{U} \subset \dot{M}^{\varepsilon_{1}} \subset \dot{M}^{a}
$$

We fix such $\varepsilon_{0}$ and $\varepsilon_{1}$.

For a compact set $K \subset \dot{M}^{a}$, since $\max f(K)<a$ holds, there exists $b$ such that $\max f(K)<b<a$. This implies that $K \subset M^{b}$.

By assumption, there are no critical points in $f^{-1}((m, a])$. Hence there is a diffeomorphism

$$
\varphi:\left(M^{\varepsilon_{1}}, M^{\varepsilon_{0}}\right) \stackrel{\cong}{\longrightarrow}\left(M^{a}, M^{b}\right) .
$$

We set $V:=\varphi(U)$. Then $V$ is an open set of $M^{a}, K \subset V$ and $V \cong \mathbb{R}^{n}$

Lemma 2. The manifold $f^{-1}(a)$ is homotopy equivalent to $S^{n-1}$.

Proof. Since $f^{-1}(a)$ is a manifold, the assertion is clear for $n=$ 2 . We assume $n \geq 3$. We claim that

$$
H_{q}\left(f^{-1}(a) ; \mathbb{Z}\right) \cong H_{q}\left(S^{n-1} ; \mathbb{Z}\right) \quad \forall q .
$$

To prove (5), note that $M^{a}$ is a manifold with boundary such that $\partial M^{a}=f^{-1}(a)$. Lefschetz duality implies that

$$
H_{q}\left(M^{a}, \partial M^{a} ; \mathbb{Z}\right) \cong H^{n-q}\left(M^{a} ; \mathbb{Z}\right) \quad \forall q .
$$

Recall that the inclusion $i: \dot{M}^{a} \rightarrow M^{a}$ is a homotopy equivalence. Since $M^{a} \cong \mathbb{R}^{n}$ by Lemma $1, M^{a}$ has the cohomology of a point. In the homology long exact sequence of the pair $\left(M^{a}, \partial M^{a}\right)$, we apply (6). Then we obtain (5).

Since the fundamental group at infinity of $M^{a}$ is trivial by Lemma $1, f^{-1}(a)$ is simply connected. (See, e.g., [3, p. 389] or [4, Examples 5 and 6] for this kind of argument.) Hence $f^{-1}(a)$ is a homotopy sphere.

Proof of Theorem A. For $n=2$ or 3, Lemma 2 immediately implies that $f^{-1}(a)$ is diffeomorphic to $S^{n-1}$.

For $n \geq 6$, Lemma 2 tells us that $M^{a}$ is contractible with simply connected boundary. Using the $h$-cobordism theorem (see [5, p. 108, Proposition A]), we have $M^{a} \cong D^{n}$. Hence $f^{-1}(a) \cong S^{n-1}$.

For $n=5$, combining Freedman's resolution of the 4dimensional Poincaré conjecture [6] and Lemma 2, we have $f^{-1}(a) \approx S^{4}$.

For $n=4$, combining Perelman's resolution of the 3dimensional Poincaré conjecture [7], Lemma 2, and the fact [8] that the differential structure on $S^{3}$ is unique, we have $f^{-1}(a) \cong S^{3}$.

Proof of Corollary B. If $f$ satisfies the assumption of Corollary $\mathrm{B}$, the function $g:=-f$ satisfies the assumption of $f$ in Theorem A. Hence Corollary B follows.

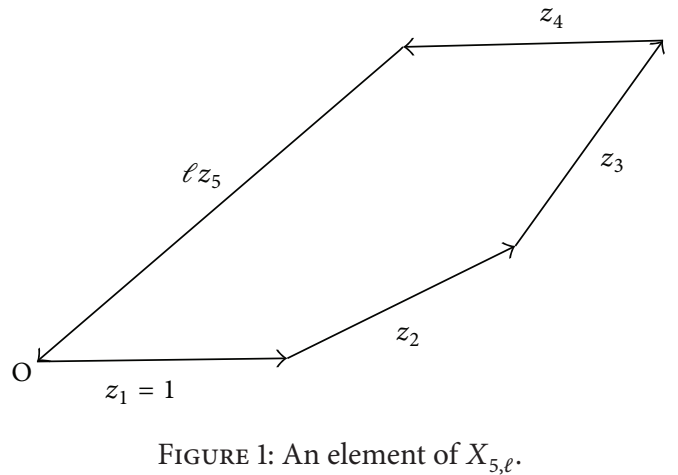

\section{An Application}

Starting in [9-11], the topology of the configuration space of planar polygons has been considered by many authors. We refer to [12] for an excellent exposition.

For simplicity, we consider the case that the edge lengths are $1, \ldots, 1$ and $\ell$. Let $S O(2)$ act on the $n$-dimensional torus $T^{n}=\left(S^{1}\right)^{n}$ diagonally. For $\ell>0$, we set

$$
X_{n, \ell}=\frac{\left\{\left(z_{1}, \ldots, z_{n}\right) \in T^{n} \mid\left(\sum_{i=1}^{n-1} z_{i}\right)+\ell z_{n}=0\right\}}{S O(2)} .
$$

Here $z_{i} \in S^{1} \subset \mathbb{C}$ denotes the unit vectors in the direction of the sides of a polygon.

It is clear that $X_{n, \ell}=\varnothing$ for $\ell>n-1$ and $X_{n, n-1}=$ \{one point\}. It is also known that there is a diffeomorphism

$$
X_{n, \ell} \cong S^{n-3} \text { for } n-3<\ell<n-1 .
$$

Recall that (8) can be understood Morse-theoretically. The following arguments are particularly well described in [12]: Using the $S O(2)$-action in the definition of $X_{n, \ell}$, we normalize $z_{1}$ to be 1 and write $X_{n, \ell}$ as

$$
\begin{aligned}
& X_{n, \ell} \\
& \quad=\left\{\left(z_{1}, \ldots, z_{n}\right) \in T^{n} \mid z_{1}=1,\left(\sum_{i=1}^{n-1} z_{i}\right)+\ell z_{n}=0\right\} .
\end{aligned}
$$

(See Figure 1.)

We identify $T^{n-2}=\left\{\left(z_{1}, \ldots, z_{n-1}\right) \in T^{n-1} \mid z_{1}=1\right\}$ and define a function $f_{n}: T^{n-2} \rightarrow \mathbb{R}$ by

$$
f_{n}\left(z_{1}, \ldots, z_{n-1}\right)=\left|\sum_{i=1}^{n-1} z_{i}\right|^{2}
$$

Then (9) gives an identification $X_{n, \ell}=f_{n}^{-1}\left(\ell^{2}\right)$. It is elementary to prove that an element $\left(z_{1}, \ldots, z_{n-1}\right) \in T^{n-2} \backslash f_{n}^{-1}(0)$ is a critical point of $f_{n}$ if and only if $z_{i} \in\{-1,1\}$ for all $2 \leq i \leq n-1$. All these critical points are nondegenerate and their indices are known. (See, e.g., $[9,12-14]$.) In particular, $f_{n}$ attains its maximum value at $\left(z_{1}, \ldots, z_{n-1}\right)=(1, \ldots, 1)$ and this is a nondegenerate critical point. Hence, using the Morse lemma, (8) follows. 
The configuration space of spatial polygons has also been studied by many authors. (See, e.g., $[12,14,15]$.) In this case, our object is defined by

$$
Y_{n, \ell}=\frac{\left\{\left(a_{1}, \ldots, a_{n}\right) \in\left(S^{2}\right)^{n} \mid\left(\sum_{i=1}^{n-1} a_{i}\right)+\ell a_{n}=0\right\}}{S O(3)} .
$$

Recently, motivated by chemistry, Crippen [16], Goto and Komatsu [17], and O'Hara [18] studied certain subspaces of $Y_{n, 1}$. Namely, they studied the configuration space of equilateral polygons with restriction on the splay angle of each vertex.

First, we define the angle $\alpha_{n}$ to be $(7 / 12) \pi$ and $\arccos (-1 /$ 3) as $n=5$ and $n \geq 6$, respectively. Goto and Komatsu [17] chose the angle $\alpha_{n}$ with molecular model in mind. Then they studied the space defined by

$$
\begin{aligned}
Z_{n} & =\left\{\left(a_{1}, \ldots, a_{n}\right) \in Y_{n, 1} \mid-\left\langle a_{i}, a_{i+1}\right\rangle=\cos \alpha_{n} \text { for } 1\right. \\
& \leq i \leq n-2\},
\end{aligned}
$$

where $\langle$,$\rangle denotes the standard inner product on \mathbb{R}^{3}$. The closed chains in $Z_{n}$ are equilateral polygons in $\mathbb{R}^{3}$ with $n$ vertices such that the interior angles are all equal to $\alpha_{n}$ except for the two angles at the successive vertices $O$ and $\sum_{i=1}^{n-1} a_{i}$.

The main result in [17] states that when $n=5,6,7, Z_{n}$ is a manifold homeomorphic to $S^{n-4}$. Since they use Reeb's theorem, they state their result as a homeomorphism. But actually $Z_{n}$ and $S^{n-4}$ are diffeomorphic because the differential structure on $S^{k}$ is unique for $k \leq 3$. But what is more important is that it is not known whether $Z_{n}$ is a manifold for $n \geq 8$.

Second, for all $\beta \in \mathbb{R}$, we define a space by

$$
\begin{aligned}
& W_{n}(\beta)=\left\{\left(a_{1}, \ldots, a_{n}\right) \in Y_{n, 1} \mid-\left\langle a_{i}, a_{i+1}\right\rangle\right. \\
& \quad=\cos \beta \text { for } 1 \leq i \leq n\} .
\end{aligned}
$$

Here we understand $a_{n+1}$ to be $a_{1}$. The closed chains in $W_{n}(\beta)$ are equilateral polygons in $\mathbb{R}^{3}$ with $n$ vertices such that the interior angles are all equal to $\beta$. Crippen [16] studied the topological type of $W_{n}(\beta)$ for various $\beta$ when $n=3,4,5$. The result is that $W_{n}(\beta)$ is either $\varnothing$, one point, or two points depending on $\beta$. Next, O'Hara [18] studied $W_{6}(\beta)$ for various $\beta$. The result is that $W_{6}(\beta)$ is disjoint union of a certain number of $S^{1}$ 's and points. It is to be noted that since $\operatorname{dim} Y_{n, 1}=2 n-6$, it is natural to expect that $\operatorname{dim} W_{n}(\beta)=$ $2 n-6-n=n-6$. But the above results imply that the defining equations for $W_{n}(\beta)$ do not intersect transversally when $n$ is small.

Note that the above results in [16-18] concentrate on the case for small $n$. This is understandable because imposing some conditions on the interior angles causes difficulties in computations. Nevertheless, we would like to prove some assertion which holds for general $n$. Modifying the definition of $Z_{n}$, we define a space as follows:

$$
\begin{aligned}
A_{n, \ell} & =\left\{\left(a_{1}, \ldots, a_{n}\right) \in Y_{n, \ell} \mid\left\langle a_{i}, a_{i+1}\right\rangle=0 \text { for } 1 \leq i\right. \\
& \leq n-2\} .
\end{aligned}
$$

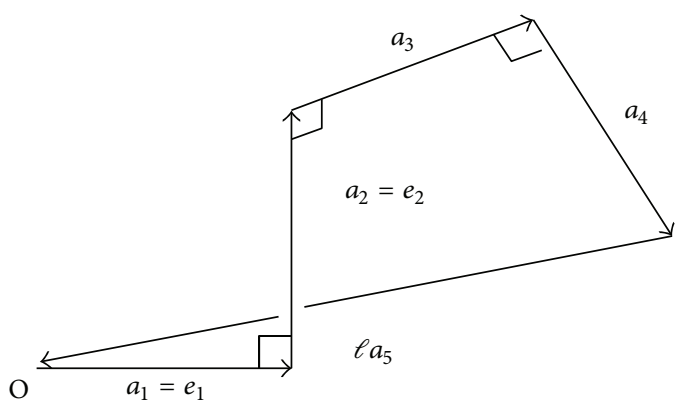

Figure 2: An element of $A_{5, \ell}$.

The closed chains in $A_{n, \ell}$ are polygons in $\mathbb{R}^{3}$ with edge lengths $\underbrace{1, \ldots, 1}_{n-1}$ and $\ell$ such that the interior angles are all equal to $\pi / 2$ except for the two angles at the endpoints of the edge of length $\ell$.

Let us obtain a similar description to (9). We set $e_{1}=$ $(1,0,0)$ and $e_{2}=(0,1,0)$. By the $S O(3)$-action in the definition of $Y_{n, \ell}$, we can normalize $a_{1}$ and $a_{2}$ to be $e_{1}$ and $e_{2}$, respectively. Thus we have the following description of $A_{n, \ell}$ :

$$
\begin{aligned}
A_{n, \ell} & =\left\{\left(a_{1}, \ldots, a_{n}\right) \in\left(S^{2}\right)^{n} \mid a_{1}=e_{1}, a_{2}\right. \\
& =e_{2}, \quad\left(\sum_{i=1}^{n-1} a_{i}\right)+\ell a_{n}=0,\left\langle a_{i}, a_{i+1}\right\rangle \\
& =0(2 \leq i \leq n-2)\} .
\end{aligned}
$$

Hereafter we use description (15). (See Figure 2.)

We recall the following.

Theorem 3 (see [19, Theorem 5.6], [20, Theorem 4]). One sets

$$
r_{n}:= \begin{cases}\sqrt{2} m & n=2 m+1 \\ \sqrt{2 m^{2}-2 m+1} & n=2 m .\end{cases}
$$

Then the following results hold:

(i) $A_{n, \ell}=\varnothing$ if $\ell>r_{n}$.

(ii) $A_{n, r_{n}}=$ \{one point $\}$. We write the element of $A_{n, r_{n}}$ by $P_{n} \stackrel{r_{n}}{=}\left(a_{1}, \ldots, a_{n}\right)$ in the notation of (15). Then $P_{n}$ is given as follows: For $1 \leq i \leq n-1, a_{i}$ is given by

$$
a_{i}= \begin{cases}e_{1} & i \text { is odd } \\ e_{2} & i \text { is even }\end{cases}
$$

As a consequence, $a_{n}$ is given by

$$
a_{n}= \begin{cases}-\frac{m}{r_{n}}\left(e_{1}+e_{2}\right) & n=2 m+1, \\ -\frac{1}{r_{n}}\left(m e_{1}+(m-1) e_{2}\right) & n=2 m .\end{cases}
$$




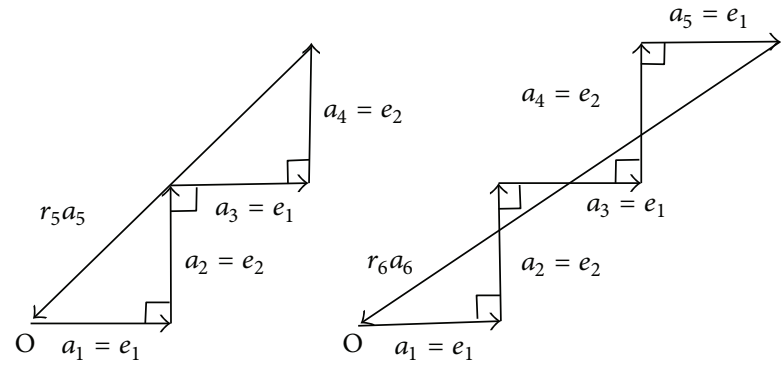

FIgURe 3: $P_{5}$ and $P_{6}$.

Now we state the main result in this section (cf. (8)).

Theorem 4. Let $\ell$ be slightly smaller than $r_{n}$. Then, for all $n \geq$ 5 , there is a diffeomorphism $A_{n, \ell} \cong S^{n-4}$.

For the rest of this paper, we prove Theorem 4. For that purpose, we define

$$
\begin{aligned}
B_{n} & =\left\{\left(a_{1}, \ldots, a_{n-1}\right) \in\left(S^{2}\right)^{n-1} \mid a_{1}=e_{1}, a_{2}\right. \\
& \left.=e_{2},\left\langle a_{i}, a_{i+1}\right\rangle=0 \quad(2 \leq i \leq n-2)\right\} .
\end{aligned}
$$

Moreover, similarly to $f_{n}$ in (10), we define a map $g_{n}: B_{n} \rightarrow$ $\mathbb{R}$ by

$$
g_{n}\left(a_{1}, \ldots, a_{n-1}\right)=\left\|\sum_{i=1}^{n-1} a_{i}\right\|^{2}
$$

Then (15) gives an identification $A_{n, \ell}=g_{n}^{-1}\left(\ell^{2}\right)$ and Theorem 3 tells us that $\max g_{n}\left(B_{n}\right)$ is attained by $P_{n}$. In order to compute the Hessian matrix of $g_{n}$ at $P_{n}$, we construct the commutative diagram shown in Figure 4.

First, we set

$$
\pi_{n}\left(\theta_{1}, \ldots, \theta_{n-3}\right)=\left(e^{i \theta_{1}}, \ldots, e^{i \theta_{n-3}}\right) .
$$

Namely, $\pi_{n}$ is the universal cover.

Second, we construct $\psi_{n}$ by induction on $n$. We define $\psi_{3}$ to be the unique map between one-point spaces.

Assuming that $\psi_{n}$ is constructed, we construct $\psi_{n+1}$. We write

$$
\psi_{n}\left(\theta_{1}, \ldots, \theta_{n-3}\right)=\left(a_{1}, \ldots, a_{n-1}\right) .
$$

Since $\left\langle a_{n-2}, a_{n-1}\right\rangle=0$, there are two choices for $\xi \in S^{2}$ which satisfies that $\left\langle\xi, a_{n-2}\right\rangle=\left\langle\xi, a_{n-1}\right\rangle=0$. Among these $\xi$ 's, we choose the one which satisfies the condition that $\operatorname{det}\left(a_{n-2}, a_{n-1}, \xi\right)=(-1)^{n+1}$. Using this, we define

$$
\eta:=\left(\cos \theta_{n-2}\right) a_{n-2}+\left(\sin \theta_{n-2}\right) \xi .
$$

And we set

$$
\psi_{n+1}\left(\theta_{1}, \ldots, \theta_{n-3}, \theta_{n-2}\right):=\left(a_{1}, \ldots, a_{n-1}, \eta\right)
$$

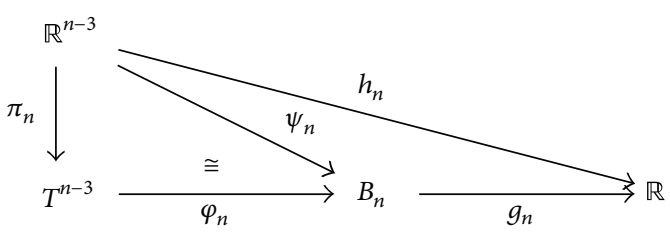

Figure 4

From the construction, $\psi_{n}$ induces a map $\varphi_{n}: T^{n-3} \rightarrow B_{n}$ such that $\psi_{n}=\varphi_{n} \circ \pi_{n}$. It is easy to see that $\varphi_{n}$ is a diffeomorphism. (See Remark 7(ii).)

Third, we set $h_{n}:=g_{n} \circ \psi_{n}$, where $g_{n}$ is defined in (20). Thus we have completed the construction of the diagram in Figure 4.

Note that $\psi_{n}(0, \ldots, 0)=P_{n}$, where $P_{n}$ is defined in Theorem 3.

Lemma 5. Let $H\left(h_{n}\right)(0, \ldots, 0)$ be the Hessian matrix of $h_{n}$ at $\left(\theta_{1}, \ldots, \theta_{n-3}\right)=(0, \ldots, 0)$. Note that this is an $(n-3) \times(n-$ 3) matrix. Let $\gamma(n, i, j)$ be the $(i, j)$ th entry of $H\left(h_{n}\right)(0, \ldots, 0)$. Then, for all $n \geq 5$, the following result holds:

$$
\gamma(n, i, j)= \begin{cases}-2\left\lfloor\frac{n-1-i}{2}\right\rfloor\left\lfloor\frac{j+1}{2}\right\rfloor & \text { if } i \geq j, \\ -2\left\lfloor\frac{n-1-j}{2}\right\rfloor\left\lfloor\frac{i+1}{2}\right\rfloor & \text { if } i<j .\end{cases}
$$

Proof. The lemma is proved by direct computations.

Proof of Theorem 4 for Even $n$. We set

$$
\begin{aligned}
\Gamma(n, i):= & \gamma(n, i, i+1) \\
& -\frac{\gamma(n, i, 1)}{\gamma(n, n-3,1)} \gamma(n, n-3, i+1) .
\end{aligned}
$$

It is easy to see that when $n$ is even,

$$
\operatorname{det}\left(H\left(h_{n}\right)(0, \ldots, 0)\right)=-2 \prod_{i=1}^{n-4} \Gamma(n, i)
$$

Thus we have $\operatorname{det}\left(H\left(h_{n}\right)(0, \ldots, 0)\right) \neq 0$; hence $(0, \ldots, 0)$ is a nondegenerate critical point of $h_{n}$. Using the Morse lemma, we complete the proof of Theorem 4 for even $n$.

Proof of Theorem 4 for Odd $n$. Lemma 5 tells us that when $n$ is odd, $\operatorname{det}\left(H\left(h_{n}\right)(0, \ldots, 0)\right)=0$. Hence we need to use Corollary B. For that purpose, it will suffice to prove that $(0, \ldots, 0)$ is an isolated critical point of $h_{n}$.

We define a map $F_{n}: S^{n-4} \times[0, \infty) \rightarrow \mathbb{R}^{n-3}$ by

$$
F_{n}\left(u_{1}, \ldots, u_{n-3}, t\right)=\left(\operatorname{grad} h_{n}\right)\left(t u_{1}, \ldots, t u_{n-3}\right) \text {, }
$$

where the right-hand side denotes the Jacobian matrix of $h_{n}$ at

$$
\left(\theta_{1}, \ldots, \theta_{n-3}\right)=\left(t u_{1}, \ldots, t u_{n-3}\right)
$$


First we prove the following.

Lemma 6. For each $\left(u_{1}, \ldots, u_{n-3}\right) \in S^{n-4}$, there exists $\varepsilon>0$ such that $F_{n}\left(u_{1}, \ldots, u_{n-3}, t\right) \neq 0$ for all $t \in(0, \varepsilon)$.

Proof. We fix $\left(u_{1}, \ldots, u_{n-3}\right) \in S^{n-4}$. In order to prove the lemma by contradiction, assume that $t=0$ were an accumulation point of $F_{n}\left(u_{1}, \ldots, u_{n-3}, t\right)$. For $1 \leq k \leq n-$ 3 , let $F_{n, k}\left(u_{1}, \ldots, u_{n-3}, t\right)$ be the $k$ th element of $F_{n}\left(u_{1}, \ldots\right.$, $\left.u_{n-3}, t\right)$. Since $F_{n, k}\left(u_{1}, \ldots, u_{n-3}, t\right)$ is a polynomial in $\left\{\sin \left(t u_{i}\right)\right.$, $\left.\cos \left(t u_{i}\right) \mid 1 \leq i \leq n-3\right\}$, we can define $F_{n, k}\left(u_{1}, \ldots, u_{n-3}, t\right)$ for $t \in \mathbb{C}$ and this is a holomorphic function. If $t=0$ were an accumulation point of $F_{n}\left(u_{1}, \ldots, u_{n-3}, t\right)$, then the identity theorem would tell us that $F_{n, k}\left(u_{1}, \ldots, u_{n-3}, t\right)$ is identically 0 for all $t \in \mathbb{C}$ and for all $1 \leq k \leq n-3$.

We write the Maclaurin expansion of $F_{n, k}\left(u_{1}, \ldots, u_{n-3}, t\right)$ as

$$
\begin{aligned}
F_{n, k}\left(u_{1}, \ldots, u_{n-3}, t\right)= & \sigma_{k}\left(u_{1}, \ldots, u_{n-3}\right) t \\
& +\tau_{k}\left(u_{1}, \ldots, u_{n-3}\right) t^{3} \\
& + \text { Higher Order Terms in } t,
\end{aligned}
$$

where $\sigma_{k}\left(u_{1}, \ldots, u_{n-3}\right)$ and $\tau_{k}\left(u_{1}, \ldots, u_{n-3}\right)$ are polynomials in $u_{1}, \ldots, u_{n-3}$ of degrees 1 and 3 , respectively. Since $F_{n, k}\left(u_{1}, \ldots, u_{n-3}, t\right) \equiv 0$, we have

$$
\begin{aligned}
\sigma_{k}\left(u_{1}, \ldots, u_{n-3}\right)=\tau_{k}\left(u_{1}, \ldots, u_{n-3}\right)= & \\
& \forall 1 \leq k \leq n-3 .
\end{aligned}
$$

Then standard computations show that $u_{1}=\cdots=u_{n-3}=0$. This contradicts the assumption that $\left(u_{1}, \ldots, u_{n-3}\right) \in S^{n-4}$.

This completes the proof of Lemma 6.

Now using Lemma 6 and the continuity of the function $F_{n}$, there exists an open neighborhood $U$ of $\mathrm{O}$ in $\mathbb{R}^{n-3}$ such that if $\left(a_{1}, \ldots, a_{n-3}\right) \in U \backslash \mathrm{O}$, then

$$
\left(\operatorname{grad} h_{n}\right)\left(a_{1}, \ldots, a_{n-3}\right) \neq(0, \ldots, 0)
$$

Thus $(0, \ldots, 0)$ is an isolated critical point of $h_{n}$.

This completes the proof of Theorem 4 for odd $n$.

Remark 7. (i) Recall that Corollary B for $\operatorname{dim} M=5$ just gives a topological assertion. In order to prove Theorem 4 , we have studied the map $g_{n}: B_{n} \rightarrow \mathbb{R}$. Since $\operatorname{dim} B_{n}=n-3$, $\operatorname{dim} B_{n}=5$ implies that $n=8$. But we have seen in Lemma 5 that when $n$ is even, $P_{n}$ is a nondegenerate critical point and deduced Theorem 4 directly from the Morse lemma, which gives a differential assertion.

(ii) Although we have not used the diffeomorphism $\varphi_{n}$ in the above arguments, it is to be noted that the map $g_{n} \circ \varphi_{n}$ : $T^{n-3} \rightarrow \mathbb{R}$ is much more difficult than the known map $f_{n}$ : $T^{n-2} \rightarrow \mathbb{R}$ in (10).

\section{Conflict of Interests}

The author declares that there is no conflict of interests regarding the publication of this paper.

\section{Acknowledgment}

The author is grateful to the referee for valuable comments.

\section{References}

[1] J. Milnor, Morse Theory, Princeton University Press, Princeton, NJ, USA, 1963.

[2] J. Milnor, "Differential topology," in Lectures on Modern Mathematics, Vol. II, pp. 165-183, John Wiley \& Sons, New York, NY, USA, 1964.

[3] M. W. Davis, "Nonpositive curvature and reflection groups," in Handbook of Geometric Topology, pp. 373-422, North-Holland, Amsterdam, The Netherlands, 2002.

[4] C. R. Guilbault, "Ends, shapes, and boundaries in manifold topology and geometric group theory," in Topology and Geometric Group Theory (Proceedings of the OSU Special Year 2010-2011), Lecture Notes in Mathematics, Springer, 2010-2011, http://arxiv.org/pdf/1210.6741v3.pdf.

[5] J. Milnor, Lectures on the h-cobordism Theorem, Princeton University Press, Princeton, NJ, USA, 1965.

[6] M. H. Freedman, "The topology of four-dimensional manifolds," Journal of Differential Geometry, vol. 17, no. 3, pp. 357453, 1982.

[7] J. W. Morgan and G. Tian, Ricci Flow and the Poincaré Conjecture, vol. 3 of Clay Mathematical Monographs, American Mathematical Society, 2007.

[8] E. E. Moise, "Affine structures in 3-manifolds. V. The triangulation theorem and Hauptvermutung," Annals of Mathematics. Second Series, vol. 56, pp. 96-114, 1952.

[9] J.-C. Hausmann, "Sur la topologie des bras articulés," in Algebraic Topology Poznan 1989, vol. 1474 of Lecture Notes in Mathematics, pp. 146-159, Springer, 1989.

[10] M. Kapovich and J. Millson, "On the moduli space of polygons in the Euclidean plane," Journal of Differential Geometry, vol. 42, no. 2, pp. 430-464, 1995.

[11] K. Walker, Configuration spaces of linkages [Undergraduate thesis], Princeton University, 1985.

[12] M. Farber, Invitation to Topological Robotics, Zurich Lectures in Advanced Mathematics, European Mathematical Society (EMS), Zürich, Switzerland, 2008.

[13] M. Farber and D. Schütz, "Homology of planar polygon spaces," Geometriae Dedicata, vol. 125, pp. 75-92, 2007.

[14] R. J. Milgram and J. C. Trinkle, "The geometry of configuration spaces for closed chains in two and three dimensions," Homology, Homotopy and Applications, vol. 6, no. 1, pp. 237-267, 2004.

[15] M. Kapovich and J. J. Millson, "The symplectic geometry of polygons in the Euclidean space," Journal of Differential Geometry, vol. 44, no. 3, pp. 479-513, 1996.

[16] G. M. Crippen, "Exploring the conformation space of cycloalkanes by linearized embedding," Journal of Computational Chemistry, vol. 13, no. 3, pp. 351-361, 1992.

[17] S. Goto and K. Komatsu, "The configuration space of a model for ringed hydrocarbon molecules," Hiroshima Mathematical Journal, vol. 42, no. 1, pp. 115-126, 2012. 
[18] J. O'Hara, “The configuration space of equilateral and equiangular hexagons," Osaka Journal of Mathematics, vol. 50, no. 2, pp. 477-489, 2013.

[19] N. Benbernou, Fixed-angle polygonal chains: locked chains and the maximum span [Undergraduate thesis], Smith College, Northampton, Mass, USA, 2006, http://people.csail.mit.edu/ nbenbern/thesis.pdf.

[20] N. Benbernou and J. O'Rourke, "On the maximum span of fixed-angle chains," in Proceedings of the 18th Canadian Conference on Computational Geometry, pp. 14-16, Kingston, Canada, August 2006, http://people.csail.mit.edu/nbenbern/ MaxSpan.CCCG.pdf. 


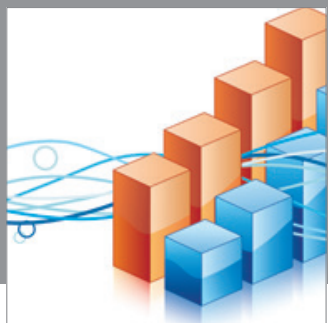

Advances in

Operations Research

mansans

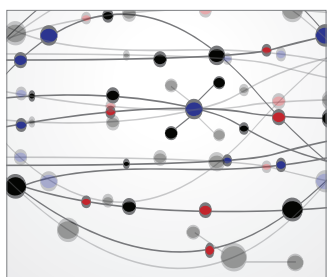

The Scientific World Journal
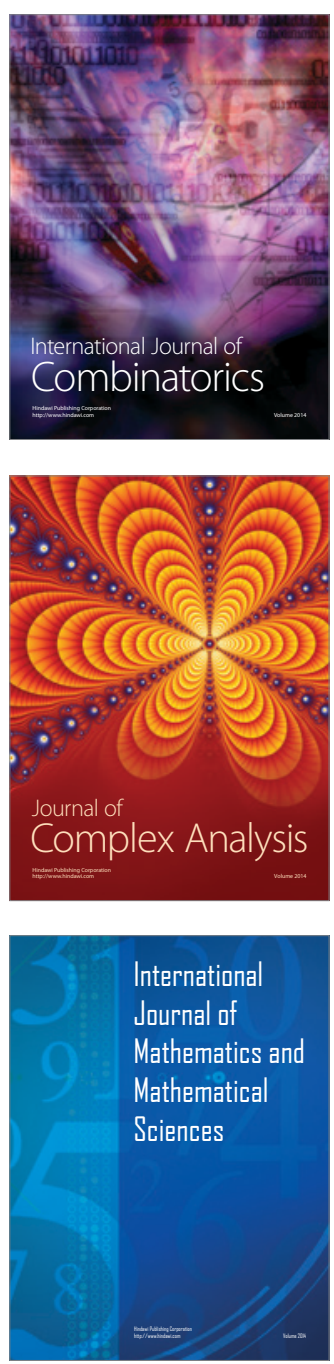
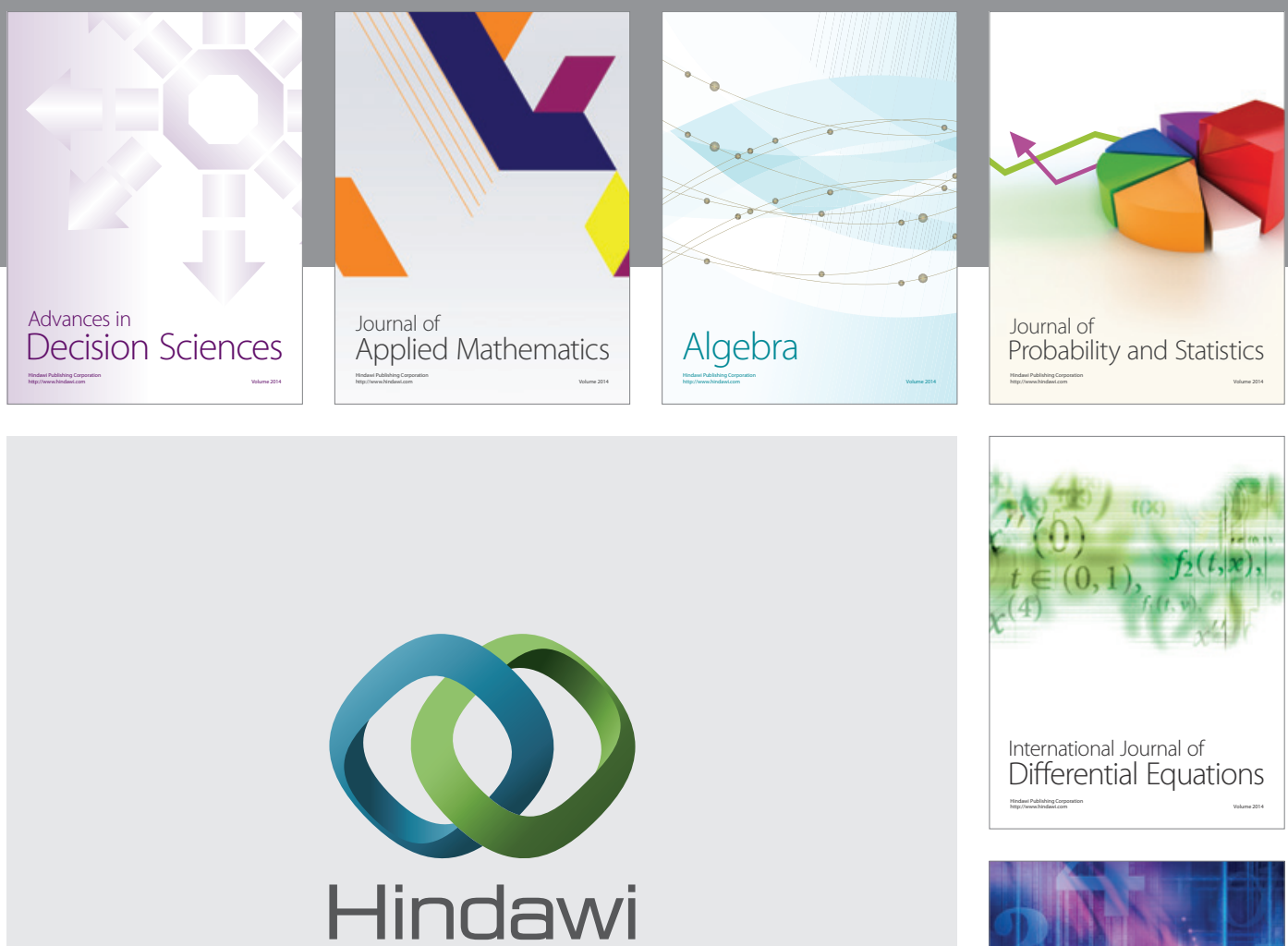

Submit your manuscripts at http://www.hindawi.com
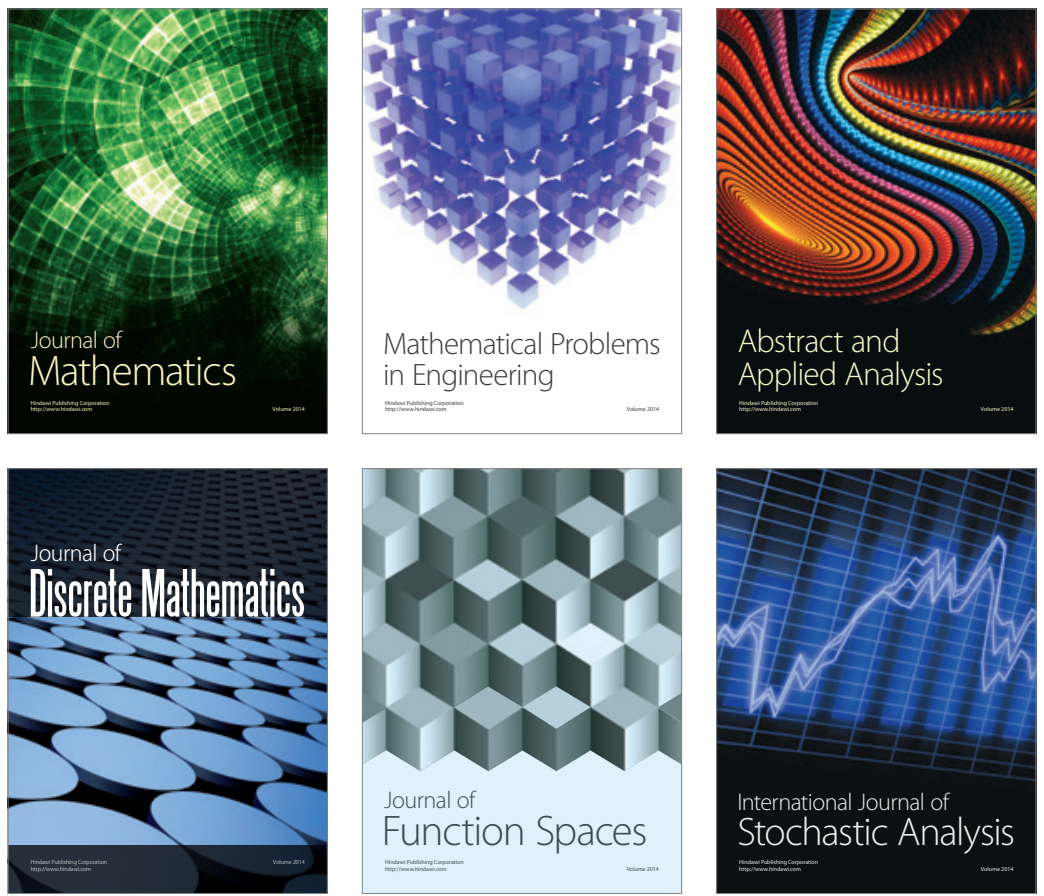

Journal of

Function Spaces

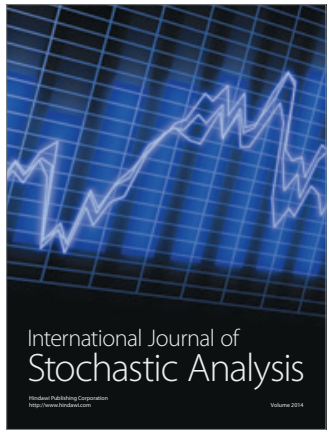

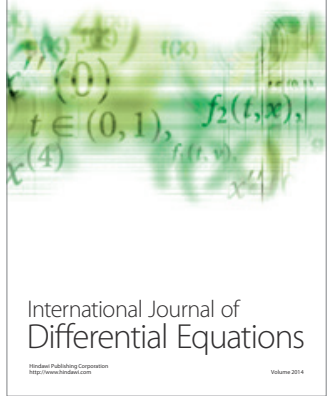
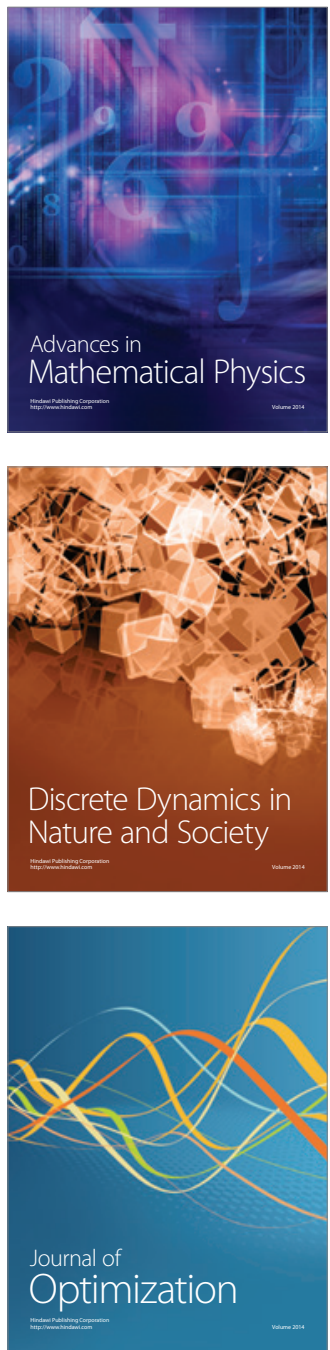